TITLE:

HIV-1 tracing method of systemic viremia in vivo using an artificially mutated virus pool( Dissertation_全文)

$\operatorname{AUTHOR}(\mathrm{S})$ :

Soper, Andrew James

\title{
CITATION:
}

Soper, Andrew James. HIV-1 tracing method of systemic viremia in vivo using an artificially mutated virus pool. 京都大学, 2021, 博士(医科学)

ISSUE DATE:

2021-03-23

URL:

https://doi.org/10.14989/doctor.k23111

RIGHT:

許諾条件により本文は2021-11-24に公開 
Kei Sato ORCID iD: 0000-0003-4431-1380

\section{HIV-1 tracing method of systemic viremia in vivo using an artificially mutated virus pool}

Andrew Soper ${ }^{1}$, Yoshio Koyanagi ${ }^{1}$, Kei Sato ${ }^{2 *}$

${ }^{1}$ Laboratory of Systems Virology, Institute for Frontier Life and Medical

Sciences, Kyoto University, Kyoto, Japan

${ }^{2}$ Division of Systems Virology, Department of Infectious Disease Control, International Research Center for Infectious Diseases, Institute of Medical Science, The University of Tokyo, Tokyo, Japan

*Correspondence: Kei Sato, Division of Systems Virology, Department of Infectious Disease Control, International Research Center for Infectious Diseases, Institute of Medical Science, The University of Tokyo, 4-6-1

Shirokanedai, Minato-ku, 1088639 Tokyo, Japan. Phone: +81-3-6409-2212. Fax: +81-3-6409-2213. E-mail: KeiSato@g.ecc.u-tokyo.ac.jp

Short title: Trace of systemic HIV-1 infection

Abstract: 134 words

\section{List of abbreviations}

AUC, area under the curve;

BM: bone marrow;

This article has been accepted for publication and undergone full peer review but has not been through the copyediting, typesetting, pagination and proofreading process, which may lead to differences between this version and the Version of Record. Please cite this article as doi: 10.1111/1348-0421.12862.

This article is protected by copyright. All rights reserved. 
cART, combination antiretroviral therapy;

dpi, days postinfection;

fDC, follicular dendritic cell;

HIV-1, human immunodeficiency virus type 1;

HSC, hematopoietic stem cell;

LN, lymph node;

MNC, mononuclear cell;

ORF, open reading frame;

PB, peripheral blood;

PBMC, peripheral blood mononuclear cell;

PHA, phytohemagglutinin;

SLO, secondary lymphoid organ;

$\mathrm{TCID}_{50,}, 50 \%$ tissue culture infective dose;

VOA, viral outgrowth assay;

WT, wild-type;

wpi, weeks postinfection

\begin{abstract}
The appearance of human immunodeficiency virus type 1 (HIV-1) plasma viremia is associated with progression to symptomatic disease and $\mathrm{CD} 4^{+} \mathrm{T}$ cell depletion. To locate the source of systemic viremia, this study employed a novel method to trace HIV-1 infection in vivo. We created JRCSF $\xi$ nef, a pool of infectious HIV-1 (strain JR-CSF) with highly mutated nef gene regions by random mutagenesis PCR and infected this mutated virus pool into both Jurkat-CCR5 cells and hematopoietic stem cell-transplanted humanized mice. Infection resulted in systemic plasma viremia in humanized mice and viral RNA sequencing helped us to identify multiple lymphoid organs such as spleen, lymph nodes and bone marrow but not peripheral blood cells as the
\end{abstract}

This article is protected by copyright. All rights reserved. 
source of systemic viremia. Our data suggests that this method could be useful for the tracing of viral trafficking in vivo.

\section{Keywords}

HIV-1; humanized mouse; source of systemic viremia

\section{Introduction}

Currently, combination antiretroviral therapy (cART) efficiently suppresses plasma viremia during human immunodeficiency virus type 1 (HIV-1) infection in the majority of infected individuals $(1,2)$. However upon interruption of cART, viral load swiftly returns indicating there are persisting infected cells (i.e., reservoirs) in the body (3-5). Thus, there is an ongoing need to understand and trace the contribution of various sources of HIV-1 plasma viremia as we continue to search for methods of eliminating residual virus in these patients.

Primary and secondary lymphoid organs such as lymph nodes (LNs), spleen, and bone marrow (BM) appear to be the most prevalent sites of HIV-1 replication as these tissues contain huge numbers of infected cells and free virions especially in the follicular dendritic cell (fDC) network of secondary lymphoid organs (6). There is substantial HIV-1 replication in $\mathrm{CD}^{+}{ }^{+} \mathrm{T}$ cells right from the beginning of infection throughout LNs despite viral loads being below the detection limit in plasma (6). This high level of HIV magnitude is maintained throughout infection as HIV-1 DNA and RNA can both be found in LN cells years after beginning CART (7-9). In addition, T cell homing to the BM is increased in HIV-1 infected patients (10) while only a low level of HIV-1 DNA and RNA is detected in peripheral blood mononuclear cells (PBMCs). The measurement of which has been aided by highly sensitive RT-PCR methods (11). Studies in rhesus macaque monkeys with simian immunodeficiency virus infections have also shown that after long periods of 
cART, locations with the highest viral DNA and RNA are the LN and spleen (12-14). In post-mortem spleen tissues of cART treated virally suppressed patients, single genome sequencing targeting the env-nef region showed that the spleen acts as a sanctuary site (15). This quantitative data strongly suggests that lymphoid organs are the source of HIV-1 systemic viremia. However, it remains unclear the extent of contribution by major lymphoid organ(s) to the source of systemic viremia in vivo. To address this, a previous study utilized barcoding and tagging tools to trace virus trafficking in vivo. Fennessey et al. generated a pool of simian immunodeficiency virus that contains $~ 9,300$ mutants with unique 34-nucleotide barcode sequences in between $v p x$ and $v p r$ genes and inoculated it into rhesus macaques to trace the rebound events after CART cessation (16). A similar method has recently been used to estimate the level of viral replication required to generate escape mutations from the cytotoxic lymphocyte response (17). However, previous studies to trace the diversity of HIV-1 in vivo and address the origin of systemic viremia have not been reported.

In this study we aimed to investigate the nature of HIV-1 compartmentalization in a hematopoietic stem cell (HSC)-transplanted humanized mice model. We attempted to trace the source of the persistent plasma viremia during untreated infection of a pool of HIV-1 containing artificially mutated nef via the sequencing of viral RNA in plasma, PBMCs, and multiple lymphoid tissues. We found that in humanized mice, the BM, LNs, and spleen can all act as the source of systemic HIV-1 viremia under untreated conditions. These tissue-resident viruses, but not peripheral blood cell-originated viruses, are deemed to have been established by and help to maintain systemic viremia.

\section{Materials and Methods}

\section{Ethics Statement}

All animal studies were conducted following the guidelines for the Care and Use of Laboratory Animals of the Ministry of Education, Culture, Sports, This article is protected by copyright. All rights reserved. 
Science and Technology, Japan. The authors received approval from the Institutional Animal Care and Use Committees (IACUC)/ethics committee of the institutional review board of Kyoto University (protocol number R0778-1). All protocols involving human subjects were reviewed and approved by the Kyoto University institutional review board. All human subjects provided written informed consent.

\section{Cells}

HEK293T cells (ATCC CRL-1573) and TZM-bl cells (obtained through the NIH AIDS Research and Reference Reagent Program) (18) were maintained in Dulbecco's modified Eagle's medium (Sigma) containing 10\% fetal calf serum, 2 mM L-glutamate (Gibco) and penicillin/streptomycin. Jurkat-CCR5 cells (19) were maintained in RPMI1640 (Sigma) containing 10\% fetal calf serum and penicillin/streptomycin.

\section{Humanized Mice}

NOD/SCID/II2rg null mice (NOG mice) (20) were obtained from the Central Institute for Experimental Animals (Kawasaki, Japan). The mice were maintained under specific pathogen-free conditions and were handled in accordance with the Regulation on Animal Experimentation at Kyoto University. Three NOG mice were irradiated by 2 days old with 10cGy/mouse of X-ray using the X-ray cabinet system (Faxitron X-ray Corporation, Tucson, $A Z$, USA). They were then intrahepatically injected with $1 \times 10^{5}$ to $2.3 \times 10^{5}$ human CD34 ${ }^{+}$HSC (isolated from human fetal liver) (21-24).

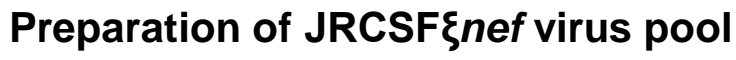

Initially three independent PCRs were carried out on separate regions of a plasmid containing full-length HIV-1 JR-CSF (pJRCSF) (GenBank accession

This article is protected by copyright. All rights reserved. 
no. M38429.1). First, the region between 5' LTR to nef upstream was amplified by conventional PCR using a high fidelity GXL DNA polymerase (Takara) with primer A (labeled in Fig. 1a) [JRCSF -91 - -54]: gag ata tac att tgg cct ttg aga gtt cat aga agg $g$ and primer B [JRCSF 8741-8772]: gcc ctt tcc aag ccc tgt ctt att ctt gta gg). pJRCSF was used as the template. The PCR product was run in gel electrophoresis and extracted. The region between nef downstream to the end of 3' LTR was also prepared using GXL DNA polymerase and primer E [JRCSF 9439-9473]: gag ctt tct aca agg gac ttc cgc tgg gga ctt $t$ and primer F [JRCSF 9781-9817]: gac agg aaa taa cca tta aga gca att cag ggg tta c). Next, a mutagenesis PCR targeting the nef region was performed using GeneMorph II Random mutagenesis kit (Agilent Technologies) and primer C [JRCSF 8741-8772]: cct aca aga ata aga cag ggc ttg gaa agg gc and primer D [JRCSF 9439-9473]: aaa gtc ccc agc gga aag tcc ctt gta gaa agc tc). pJRCSF was used as the template. The PCR product was purified and two additional mutagenesis PCRs were performed using the 1st PCR product as the template. To verify the mutation efficacy, aliquots of the PCR product at each step (1st, 2nd and 3rd steps) were cloned into TOPO vectors for sequencing ("Sequencing $A$ " in Fig. 1a). Finally, the three PCR fragments were conjugated by overlap extension PCR using KOD FX Neo DNA polymerase (Toyobo) with primers $A$ and $F$ and full-length JRCSF DNA

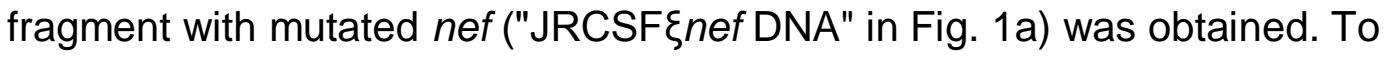
verify the extent of mutations in the nef region of JRCSF $\xi n e f$ DNA fragment, PCR was performed using GXL DNA polymerase with primers $C$ and D. The products were cloned into TOPO vectors and nef gene region of individual clones was sequenced ("Sequencing B" in Fig. 1a).

To generate a replication-competent nef-mutated JRCSF (henceforth

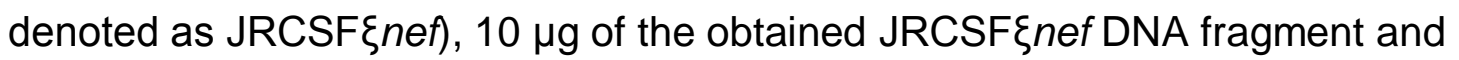
$20 \mu \mathrm{g}$ of pUC19 were cotransfected into HEK293T cells using a standard calcium phosphate protocol as previously described (25). At 48 hours posttransfection, the culture supernatant was harvested, centrifuged, and then filtered through a $0.45-\mu \mathrm{m}$-pore-size filter (Millipore). Viral infectivity was measured with TZM-bl cells and the $50 \%$ tissue culture infective dose $\left(T_{C} I_{50}\right)$ of standard virus was measured by Reed-Muench's method as

This article is protected by copyright. All rights reserved. 
previously described (26). Briefly, serially diluted JRCSF $\xi$ nef virus pool and the standard virus (JR-CSF) were inoculated into TZM-bl cells and infectivity was measured by TZM-bl assay $(25,21)$. Infectivity was $52,684 \mathrm{TCID}_{50} / \mathrm{ml}$.

\section{HIV-1 Infection}

For the experiments in in vitro cell culture (Fig. 2), 100,000 Jurkat-CCR5 cells were infected with a JRCSF $\xi n e f\left(1,000 \mathrm{TCID}_{50}\right.$; multiplicity of infection 0.01$)$. The culture supernatant was harvested every two days (i.e., 2, 4, 6, 8, 10, 12 and 14 days post infection [dpi]) for sequencing of the nef region (at 6,10 and $14 \mathrm{dpi}$ ) and measuring viral RNA level. During harvest of the culture supernatant, culture media was replaced with fresh media. These in vitro experiments were performed in four independent cultures. The nef gene region from viruses in culture supernatant at 6,10 and 14 dpi were sequenced and combined together on a phylogeny tree for each culture. 70, 83, 63 and 76 sequences were isolated and sequenced from cultures A-D respectively. For the experiments in humanized mice, (Fig. 3), 10,000 TCID 50 of JRCSF $\xi$ nef was injected intraperitoneally into three humanized mice between 12-13 weeks post-HSC transplantation.

\section{RNA extraction, cDNA preparation and Sanger sequencing}

Viral RNA was extracted from the culture supernatant or the plasma of infected humanized mice using QIAamp viral RNA mini kit (Qiagen). cDNA was synthesized with SuperScript III reverse transcriptase (Thermo Fisher Scientific) and primer D as previously described (27). cDNA was cloned into TOPO vectors and the nef region of individual clones were sequenced.

This article is protected by copyright. All rights reserved. 


\section{Collection of peripheral blood (PB) and quantification HIV-1 RNA in plasma from humanized mice}

PB was collected from humanized mice at 1, 2, 4, 6 and 9 weeks postinfection (wpi) as described previously (25). Red blood cells in the PB were lysed with $1 \times$ BD Lysis Buffer (BD Pharmingen) and the copy number of viral RNA in plasma was measured routinely by Bio Medical Laboratories Inc, Tokyo, Japan.

\section{Quantification of $\mathrm{CD4}{ }^{+} \mathrm{T}$ cell decrease in PB}

Flow cytometry was performed with a FACSCalibur (BD Biosciences) as previously described (22-24) and the obtained data was analyzed with CellQuest software (BD Biosciences) and FlowJo software (Tree Star, Inc.). For flow cytometry analysis, the following antibodies were used: PEconjugated anti-CD45 antibody (HI30; Biolegend), FITC-conjugated anti-CD3 antibody (UCHT1; Biolegend), and APC-conjugated anti-CD4 antibody (RPAT4; Biolegend).

\section{Isolation of human mononuclear cells (MNCs) from organs}

Infected mice were euthanized with $500 \mu \mathrm{L}$ of $1.2 \%$ avertin (Sigma-Aldrich) prepared in saline $(0.2 \mathrm{ml} / 10 \mathrm{~g}$ body weight) at 9 wpi. Human MNCs from BM, spleen, and LNs were collected using Ficoll-Paque (Pharmacia) and stored at $-80^{\circ} \mathrm{C}$ until use.

\section{Molecular phylogenetic analyses}

Fasta files were made in TextEdit and aligned in MEGA6 software (28). Using MEGA6, primary phylogeny trees were constructed via the maximum likelihood method before transferring to FigTree (version 1.4.3) for final phylogeny tree construction. 


\section{Viral outgrowth assay (VOA)}

Human peripheral blood mononuclear cells (PBMCs) were separated using Ficoll-Paque (Pharmacia) and then stimulated with phytohemagglutinin (PHA; Sigma) and recombinant human IL-2 (100 unit/ml) for $\sim 1$ week. PHA-activated PBMCs $\left(5 \times 10^{5}\right.$ cells) were co-cultured for 14 days with $5 \times 10^{5}$ splenic MNCs obtained from infected humanized mice. Supernatant was collected at $8 \mathrm{dpi}$ and viral RNA was isolated from culture supernatant as described above.

\section{Results}

\section{Generation of a replication-competent HIV-1 pool with highly mutated nef}

To create a mutant virus pool that is able to replicate at a similar level to wildtype (WT), random mutagenesis PCR was performed on the nef ORF of HIV1 (strain JR-CSF), with primers C and D (see "Materials and Methods" and Fig. 1a) using a low fidelity DNA polymerase. "Sequencing A" in Fig. 1a shows the creation of a sequentially greater number of nucleotide mutations in the nef gene product pool from 1st, 2nd and 3rd mutagenesis PCR, respectively (Supplementary Fig. 1 and 2). To prepare JRCSF $\xi$ nef DNA, a library of full-length HIV-1 DNA with highly mutated nef regions, the mutated nef products were conjugated to the downstream and upstream remainders of the HIV-1 sequence (Fig. 1b). Sanger sequencing verified the nef region of JRCSF $\xi$ nef DNA contained a high number of mutations ("Sequencing $B$ " in Fig. 1a and Supplementary Fig. 3). We then produced infectious HIV-1 particles via transfection of the JRCSF $\xi$ nef DNA fragment. The culture supernatant of transfected cells then contained infectious viruses (Fig. 1c),

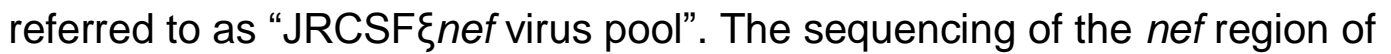

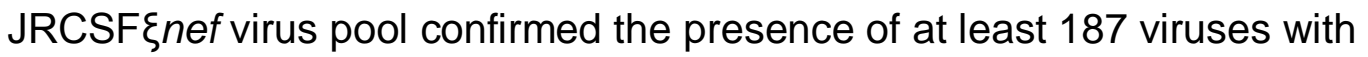
unique nef sequences and 9 viruses with WT nef amongst the 270 clones sequenced (Supplementary table 1). The nef region of JRCSF $\xi$ nef virus pool varied anywhere between 1 mutation (23/270) to more than 10 mutations This article is protected by copyright. All rights reserved. 
(34/270) (Fig. 1d and Supplementary table 1) and was highly diverse (Fig. $1 \mathrm{e})$.

\section{JR-CSFॄnef virus pool exhibits random predomination of mutant virus infection in Jurkat-CCR5 cells}

To investigate the replication dynamics of JRCSF $\xi$ nef virus pool in in vitro cell

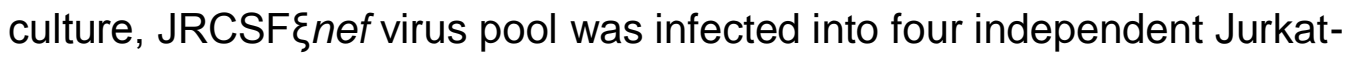
CCR5 cell cultures. As shown in Fig. 2a, the JRCSF $\xi$ nef virus pool was replication competent. Upon analyzing viral sequences, 25, 36, 15 and 19 unique sequences were independently found in cultures $A-D$. Only culture $C$ had 1 WT nef sequence at $10 \mathrm{dpi}$. The L86S/G109A (in amino residues) mutant virus predominated in 3 out of the 4 ( $A, B$ and $C$ ) cultures making up $30 \%, 33 \%, 27 \%$ and $9 \%$ of viruses in cultures $A-D$. No other viruses were in common between the 4 independent cultures (Fig. 2b). Interestingly, the viruses that dominantly replicated in each culture were different: the A36A/A70A/F78S/L86L mutant predominated only in culture D (Fig. 2b). These observations suggest that the viruses dominantly replicating in in vitro cell culture were stochastically selected.

\section{JR-CSFほnef virus pool shows systemic replicative fitness with significant pathogenicity}

To analyze the replication and pathogenic potential of JRCSF $\xi$ nef virus pool in an in vivo animal model of HIV-1, we used humanized mice. Blood samples were taken at 1, 2, 4 and 6 wpi and viral RNA was isolated from the plasma. Mice were sacrificed at 9 wpi and intracellular viral RNA was isolated from BM (both left and right femurs), spleen, PBMCs and mesenteric LNs (Fig. 3a).

JRCSF $\xi$ nef virus pool and WT JRCSF were infected into three and five mice respectively, at the same infectious dose $\left(10,000 T \mathrm{TCI}_{50}\right)$. Although these small numbers of mice do not allow for any statistical significance 
during comparisons, by 1 wpi plasma viral RNA level in WT JRCSF-infected mice was 5-fold greater than that of JRCSF nef virus pool-inoculated mice and 108-fold greater by 9 wpi (Fig. 3b). Despite viral load being relatively so much lower in JRCSF nef virus pool-inoculated mice, the decrease in peripheral $\mathrm{CD} 4^{+} \mathrm{T}$ cell number was similar for both WT and JRCSF $\xi$ nef infected groups (Fig. 3c). These observations suggest that despite less efficient replication by JRCSF $\xi$ nef virus pool, this virus pool is pathogenic and results in the depletion of $\mathrm{CD} 4^{+} \mathrm{T}$ cells in humanized mice.

\section{In vivo tracing of nef-mutated HIV-1 in plasma and tissue cells indicate HIV-1 trafficking}

To address the diversity of HIV-1 in the plasma as well as the multiple organs of three mice inoculated with JRCSF nef virus pool, we extracted viral RNA and determined the sequences. As shown in Fig. $3 d$, a variety of nef mutants were detected. Since no mutations were observed in the gag region (data not shown), the majority of the mutations in the nef region detected appeared to be derived from the artificial mutagenesis (Fig. 1).

In the plasma and BM of mouse \#1, the L86S/G109A mutant virus which predominated in 3 out of the 4 Jurkat-CCR5 cell cultures was again detected. This mutant virus was found in the plasma at 1 wpi (54\%), 2 wpi (17\%), 4 wpi (56\%), and 6 wpi (85\%) as well as in the BM collected from the left femur (28\%) at 9 wpi (Fig. 3d, top). On the other hand, a WT nef virus predominated in mouse \#2. It was found in 2 separate mesenteric LNs $(26 \%$ and $100 \%$ ), BM of both femurs (53\% and 11\%), and plasma at 1, 2, 4 (all 100\%) and 9 wpi (92\%) (Fig. 3d, middle). Although the L86S/G109A mutant

was detected in mouse \#2, it was only found in the spleen at 9 wpi (33\%) (Fig. 3d, middle), suggesting that the L86S/G109A mutant is not highly replicative in vivo. Moreover, in mouse \#3, the D28G mutant virus predominated and was found in BM of both femurs (18 and 50\%), the spleen (47\%) and plasma at 4 wpi (86\%) (Fig. 3d, bottom). The R19K/D28G mutant virus was also found in the spleen (6\%) and plasma at 6 wpi (31\%), while WT viruses were found in

This article is protected by copyright. All rights reserved. 
both the spleen (29\%) and the BM of left femur (27\%) (Fig. 3d, bottom). Because the detected mutants were different across the three mice, our data suggests dominantly replicating viruses were also stochastically selected in vivo.

To trace the source(s) of systemic viremia (i.e., viruses in plasma), we compared viral sequences between plasma and lymphoid samples. As shown in Fig. 3d, identical viruses were observed in both tissue and plasma in the same mouse. In all 3 mice, an identical virus was found in both BM and plasma. An identical virus was also found in both the plasma and either mesenteric LNs or spleen in mouse \#2 and \#3, respectively. These observations suggest that BM is a mainstay source of systemic viremia and spleen and LNs may also contribute to systemic viremia in this mouse model. Interestingly, identical viruses were never found in both the plasma and PBMCs, suggesting PBMCs are not the source of plasma virus in our system.

\section{VOA from infected mice}

Finally, to address whether the nef mutant viruses detected in lymphoid tissues are replication competent, the splenic MNCs of mice \#1 and \#3 were co-cultured with PHA-stimulated human PBMCs (Fig. 4a). At 8 dpi, the culture supernatants were harvested and the nef region was sequenced. As shown in Fig. $4 \mathrm{~b}, 90 \%$ of the 20 sequences obtained from the co-culture with mouse \#1 spleen possessed the W5G mutation. In the co-culture with mouse \#3 spleen, $95 \%$ of the 20 nef sequences had the D28G mutation (Fig. 4c). Importantly, W5G and D28G were respectively detected in the spleen of mice \#1 and \#3 (Fig. 2d). Therefore, our findings show that at least W5G and D28G mutant viruses are replication competent in vitro and in vivo and our observations in nef mutations reflect the diversity of replication-competent viruses.

This article is protected by copyright. All rights reserved. 


\section{Discussion}

In this study we used a novel method to trace the nature of systemic HIV-1 trafficking in an in vivo mouse system using a replication-competent HIV-1 pool with artificially mutated nef. We traced the source of the persistent plasma viremia and found that BM and secondary lymphoid organs (SLOs; e.g., spleen, LNs and gut-associated lymphoid tissues) but not PB are major compartments of HIV-1 virions that systemically circulate in the plasma in vivo.

Neither the L86S/G109A mutant virus, which predominated in both three of four of the Jurkat-CCR5 cultures and in mouse \#1, nor the D28G mutant virus that predominated in mouse \#3, were observed in the input JRCSF nef virus pool. Because the L86S/G109A mutant was detected both in vitro (Fig. 2) and in vivo (Fig. 3), we believe the most likely possibility is that this mutant was already present but not detected in the initial 270 sequences screened in the input pool. To fully elucidate the diversity of the JRCSF $\xi$ nef virus pool, deep sequencing using next generation sequencing technology will be needed.

It is also important to consider the large difference in time span between the three stages of these experiments. The input virus was generated in HEK293T cells over 48 hours (Fig. 1), the viruses were collected from Jurkat-CCR5 cell cultures for 2 weeks (Fig. 2) and blood was taken from humanized mice until 9 weeks (Fig. 3). Therefore, we must acknowledge when comparing virus sequences between these different systems that time spans are significantly longer in the third stage of experiments in humanized mice (Fig. 3). Because of the differential and more diverse selective intrinsic pressures present in a mouse compared with those in Jurkat-CCR5 cell monoculture, comparisons between these systems have a limited meaning.

In multiple studies the majority of HIV-1 replication has been observed to occur in SLOs $(6,12,15,13,14)$ perhaps enlightening us as to why such a high number of unique viruses were observed in the spleen of our mice. 
Unfortunately, mesenteric LNs were only recovered in one mouse so we cannot observe any trend in LNs.

HIV-1 RNA ${ }^{+}$cells are known to concentrate in B cell follicles of SLOs $(29,30)$ and $C D 8^{+} T$ cells play an important role in suppressing viral replication in both SIV models and HIV-1 infected individuals (31-33). This is mostly in extrafollicular areas as the B cell follicle appears to be a somewhat immune privileged zone. However, it is difficult to reproduce the induction of $\mathrm{CD}^{+} \mathrm{T}$ cell response in HSC transplanted humanized mice with HIV-1 infection (24). Therefore adaptive immunological responses against HIV-1 seem to be weak in our humanized mice, and immune privileged zones in $\mathrm{B}$ cell follicles are probably not the reason for spleen and LNs acting as the source of plasma virus in two of three mice. In mouse \#1, the L86S/G109A mutant virus is prevalent at all time points in plasma except at 9 wpi $(54 \%$, $17 \%, 56 \%$ and $85 \%$ in weeks 1, 2, 4 and 6 plasma), as well as making up $28 \%$ of BM viruses although was not observed in PBMCs or spleen. Its presence in the BM however suggests it could be seeded into the bloodstream again at any time point. In mouse \#2 a similar phenomenon is observed where WT virus predominates in plasma even until time of sacrifice. Although both BM and LNs still contain WT virus [26\% in LN1, 100\% in LN2, $53 \%$ in BM (left) and $11 \%$ in BM (right)]; the spleen, LN1, both BM and PBMCs at sacrifice all consist of a range of unique viruses relative to the plasma at earlier time points. In mouse \#3, we see the constant presence of mutant D28G virus, making up $41 \%$ and $84 \%$ of plasma viruses at weeks 4 and 9 and almost $50 \%$ of viruses in two of three lymphoid organs [ spleen and BM (right)] at sacrifice.

The W5G virus in mouse \#1 was found to make up $90 \%$ of total viruses in the VOA despite only making up $4 \%(1 / 23)$ of the viral RNA sequences identified in the spleen. This may suggest that the majority of splenic viral RNA sequences are not replication competent, while the W5G virus clearly is. The D28G virus in mouse \#3 was also confirmed to be replication competent in the VOA, suggesting these mutations provides some sort of replicative 
advantage. To the best of our knowledge, there has been no reports thus far of the mutations D28G, L86G or G109A resulting in altered Nef function.

In general, we observed BM and SLOs to contain a much greater range of unique viruses at 9 wpi sacrifice than in plasma. In the plasma of all mice from week 6 onwards, there was just one or two dominant sequences that made up $>92 \%$ of all viruses, suggesting these viruses have the highest replication fitness in the plasma. The observation in mouse \#1 that there was a much higher number of unique viruses in the spleen relative to plasma or even BM and PBMCs, while in mouse \#2; BM, SLOs and PBL and in mouse $\# 3$, BM and spleen always had the highest numbers of unique viruses; supports previous findings that replication may proceed with less selective pressure in SLOs and BM. Although, unlike in humans where lack of cytotoxic T lympocytes in the B cell follicle of SLOs may contribute to such an observation, the reason for increased range of viruses in these compartments in humanized mice remains unknown.

In conclusion, we have used a novel method to successfully trace HIV1 across various lymphoid organs and plasma. We identified both primary (BM) and secondary (LNs and spleen) lymphoid organs as the source of systemic HIV-1 viremia in our humanized mice. We think these findings show this method to be useful for further viral dynamics studies under CART conditions, as well as the reliability of these humanized mice to reflect scenarios occurring in human HIV-1 infections.

\section{Acknowledgments}

We would like to thank Naoko Misawa (Institute for Frontier Life and Medical Sciences, Kyoto University, Japan) and Yoriyuki Konno (Institute of Medical Science, the University of Tokyo, Japan), Tsuzumi Tsukada and Kazuho Higuchi (Niigata University, Japan) for technical support.

This study was supported in part by AMED Research Program on HIV/AIDS $19 f k 0410014$ (to Y.K. and K.S.), $19 f k 0410019$ (to K.S.); Research

This article is protected by copyright. All rights reserved. 
Program on Emerging and Re-emerging Infectious Diseases $20 \mathrm{fk} 0108146$ (to K.S.), $19 f k 0108171$ (to K.S.), $20 f k 0108270$ (to Y.K. and K.S.); Grants-in-Aid for Scientific Research (KAKENHI) Scientific Research B $18 \mathrm{H} 02662$ (to K.S.), Scientific Research on Innovative Areas 16H06429 (to K.S.), 16K21723 (to K.S.), $17 \mathrm{H} 05813$ (to K.S.), and $19 \mathrm{H} 04826$ (to K.S.); Takeda Science Foundation (to K.S.); ONO Medical Research Foundation (to K.S.); Ichiro Kanehara Foundation (to K.S.); Lotte Foundation (to K.S.); Mochida Memorial Foundation for Medical and Pharmaceutical Research (to K.S.); Daiichi Sankyo Foundation of Life Science (to K.S.); Sumitomo Foundation (to K.S.); Uehara Foundation (to K.S.); Joint Research Project of the Institute of Medical Science, the University of Tokyo (to Y.K.); Joint Usage/Research Center program of Institute for Frontier Life and Medical Sciences, Kyoto University (to K.S.); International Joint Research Project of the Institute of Medical Science, the University of Tokyo 2020-K3003 (to K.S.).

\section{Disclosure}

All authors have no competing interests to declare.

\section{Data Availability Statement}

All data is available upon request.

\section{References}

1 Antiretroviral Therapy Cohort, C. (2017) Survival of HIV-positive patients starting antiretroviral therapy between 1996 and 2013: a collaborative analysis of cohort studies. Lancet HIV, 4: e349-e56. Robins, J.M., Egger, M. Swiss, H.I.V.C.S. (2005) Long-term effectiveness of potent antiretroviral therapy in preventing AIDS and death: a prospective cohort study. Lancet, 366: 378-84.

This article is protected by copyright. All rights reserved. 

M.A. Fauci, A.S. (1997) Presence of an inducible HIV-1 latent reservoir during highly active antiretroviral therapy. Proc Natl Acad Sci U S A, 94: 13193-7.

Finzi, D., Hermankova, M., Pierson, T., Carruth, L.M., Buck, C., Chaisson, R.E., Quinn, T.C., Chadwick, K., Margolick, J., Brookmeyer, R., Gallant, J., Markowitz, M., Ho, D.D., Richman, D.D. Siliciano, R.F. (1997) Identification of a reservoir for HIV-1 in patients on highly active antiretroviral therapy. Science, 278: 1295-300.

Wong, J.K., Hezareh, M., Gunthard, H.F., Havlir, D.V., Ignacio, C.C., Spina, C.A. Richman, D.D. (1997) Recovery of replication-competent HIV despite prolonged suppression of plasma viremia. Science, 278: 1291-5.

Pantaleo, G., Graziosi, C., Demarest, J.F., Butini, L., Montroni, M., Fox, C.H., Orenstein, J.M., Kotler, D.P. Fauci, A.S. (1993) HIV infection is active and progressive in lymphoid tissue during the clinically latent stage of disease. Nature, 362: 355-8.

Wong, J.K., Gunthard, H.F., Havlir, D.V., Zhang, Z.Q., Haase, A.T., Ignacio, C.C., Kwok, S., Emini, E. Richman, D.D. (1997) Reduction of HIV-1 in blood and lymph nodes following potent antiretroviral therapy and the virologic correlates of treatment failure. Proc Natl Acad Sci U S A, 94: 12574-9. resistance in lymph node mononuclear cell RNA despite effective HAART. AIDS, 15: 1965-9.

Gunthard, H.F., Havlir, D.V., Fiscus, S., Zhang, Z.Q., Eron, J., Mellors, J., Gulick, R., Frost, S.D., Brown, A.J., Schleif, W., Valentine, F., Jonas, L., Meibohm, A., Ignacio, C.C., Isaacs, R., Gamagami, R., Emini, E., Haase, A., Richman, D.D. Wong, J.K. (2001) Residual human immunodeficiency virus (HIV) Type 1 RNA and DNA in lymph nodes and HIV RNA in genital secretions and in cerebrospinal fluid after suppression of viremia for 2 years. $J$ Infect Dis, 183: 1318-27.

Chen, J.J., Huang, J.C., Shirtliff, M., Briscoe, E., Ali, S., Cesani, F., Paar, D. Cloyd, M.W. (2002) CD4 lymphocytes in the blood of HIV(+) individuals migrate rapidly to lymph nodes and bone marrow: support for homing theory of CD4 cell depletion. J Leukoc Biol, 72: 271-8.

Pasternak, A.O., Adema, K.W., Bakker, M., Jurriaans, S., Berkhout, B., Cornelissen, M. Lukashov, V.V. (2008) Highly sensitive methods based on seminested real-time reverse transcription-PCR for quantitation of human immunodeficiency virus type 1 unspliced and multiply spliced RNA and proviral DNA. J Clin Microbiol, 46: 2206-11.

North, T.W., Higgins, J., Deere, J.D., Hayes, T.L., Villalobos, A., Adamson, L., Shacklett, B.L., Schinazi, R.F. Luciw, P.A. (2010) Viral sanctuaries during highly active antiretroviral therapy in a nonhuman primate model for AIDS. J Virol, 84: 2913-22.

Kline, C., Ndjomou, J., Franks, T., Kiser, R., Coalter, V., Smedley, J., Piatak, M., Jr., Mellors, J.W., Lifson, J.D. Ambrose, Z. (2013) Persistence of viral reservoirs in multiple tissues after antiretroviral therapy suppression in a macaque RT-SHIV model. PLoS One, 8: e84275.

Horiike, M., Iwami, S., Kodama, M., Sato, A., Watanabe, Y., Yasui, M., Ishida, Y., Kobayashi, T., Miura, T. Igarashi, T. (2012) Lymph nodes harbor viral reservoirs that cause rebound of plasma viremia in SIV-infected macaques upon cessation of combined antiretroviral therapy. Virology, 423: 107-18.

This article is protected by copyright. All rights reserved. 
Fennessey, C.M., Pinkevych, M., Immonen, T.T., Reynaldi, A., Venturi, V., Nadella, P., Reid, C., Newman, L., Lipkey, L., Oswald, K., Bosche, W.J., Trivett, M.T., Ohlen, C., Ott, D.E., Estes, J.D., Del Prete, G.Q., Lifson, J.D., Davenport, M.P. Keele, B.F. (2017) Genetically-barcoded SIV facilitates enumeration of rebound variants and estimation of reactivation rates in nonhuman primates following interruption of suppressive antiretroviral therapy. PLoS Pathog, 13: e1006359. Immonen, T.T., Camus, C., Reid, C., Fennessey, C.M., Del Prete, G.Q., Davenport, M.P., Lifson, J.D. Keele, B.F. (2020) Genetically barcoded SIV reveals the emergence of escape mutations in multiple viral lineages during immune escape. Proc Natl Acad Sci U S A, 117: 494-502.

Wei, X., Decker, J.M., Liu, H., Zhang, Z., Arani, R.B., Kilby, J.M., Saag, M.S., Wu, X., Shaw, G.M. Kappes, J.C. (2002) Emergence of resistant human immunodeficiency virus type 1 in patients receiving fusion inhibitor (T-20) monotherapy. Antimicrob Agents Chemother, 46: 1896-905.

Yamada, E., Nakaoka, S., Klein, L., Reith, E., Langer, S., Hopfensperger, K., Iwami, S., Schreiber, G., Kirchhoff, F., Koyanagi, Y., Sauter, D. Sato, K. (2018) Human-specific adaptations in Vpu conferring anti-tetherin activity are critical for efficient early HIV-1 replication in vivo. Cell Host Microbe, 23: 110-20 e7.

Ito, M., Hiramatsu, H., Kobayashi, K., Suzue, K., Kawahata, M., Hioki, K., Ueyama, Y., Koyanagi, Y., Sugamura, K., Tsuji, K., Heike, T. Nakahata, T. (2002) NOD/SCID/gamma(c)(null) mouse: an excellent recipient mouse model for engraftment of human cells. Blood, 100: 3175-82.

Nakano, Y., Misawa, N., Juarez-Fernandez, G., Moriwaki, M., Nakaoka, S., Funo, T., Yamada, E., Soper, A., Yoshikawa, R., Ebrahimi, D., Tachiki, Y., Iwami, S., Harris, R.S., Koyanagi, Y. Sato, K. (2017) HIV-1 competition experiments in humanized mice show that APOBEC3H imposes selective pressure and promotes virus adaptation. PLoS Pathog, 13: e1006348.

Nie, C., Sato, K., Misawa, N., Kitayama, H., Fujino, H., Hiramatsu, H., Heike, T., Nakahata, T., Tanaka, Y., Ito, M. Koyanagi, Y. (2009) Selective infection of CD4+ effector memory T lymphocytes leads to preferential depletion of memory T lymphocytes in R5 HIV-1-infected humanized NOD/SCID/IL-2Rgammanull mice. Virology, 394: 64-72.

Sato, K., Izumi, T., Misawa, N., Kobayashi, T., Yamashita, Y., Ohmichi, M., Ito, M., TakaoriKondo, A. Koyanagi, Y. (2010) Remarkable lethal G-to-A mutations in vif-proficient HIV-1 provirus by individual APOBEC3 proteins in humanized mice. J Virol, 84: 9546-56.

Sato, K., Nie, C., Misawa, N., Tanaka, Y., Ito, M. Koyanagi, Y. (2010) Dynamics of memory and naive CD8+ T lymphocytes in humanized NOD/SCID/IL-2Rgammanull mice infected with CCR5-tropic HIV-1. Vaccine, 28 Suppl 2: B32-7.

Sato, K., Misawa, N., Iwami, S., Satou, Y., Matsuoka, M., Ishizaka, Y., Ito, M., Aihara, K., An, D.S. Koyanagi, Y. (2013) HIV-1 Vpr accelerates viral replication during acute infection by exploitation of proliferating CD4+ T cells in vivo. PLoS Pathog, 9: e1003812.

Sato, K., Misawa, N., Fukuhara, M., Iwami, S., An, D.S., Ito, M. Koyanagi, Y. (2012) Vpu augments the initial burst phase of HIV-1 propagation and downregulates BST2 and CD4 in humanized mice. J Virol, 86: 5000-13.

Sato, K., Takeuchi, J.S., Misawa, N., Izumi, T., Kobayashi, T., Kimura, Y., Iwami, S., TakaoriKondo, A., Hu, W.S., Aihara, K., Ito, M., An, D.S., Pathak, V.K. Koyanagi, Y. (2014) APOBEC3D and APOBEC3F potently promote HIV-1 diversification and evolution in humanized mouse model. PLoS Pathog, 10: e1004453.

This article is protected by copyright. All rights reserved. 
Tamura, K., Stecher, G., Peterson, D., Filipski, A. Kumar, S. (2013) MEGA6: Molecular Evolutionary Genetics Analysis version 6.0. Mol Biol Evol, 30: 2725-9.

Folkvord, J.M., Armon, C. Connick, E. (2005) Lymphoid follicles are sites of heightened human immunodeficiency virus type 1 (HIV-1) replication and reduced antiretroviral effector mechanisms. AIDS Res Hum Retroviruses, 21: 363-70.

Biberfeld, P., Chayt, K.J., Marselle, L.M., Biberfeld, G., Gallo, R.C. Harper, M.E. (1986) HTLV-III expression in infected lymph nodes and relevance to pathogenesis of lymphadenopathy. Am J Pathol, 125: 436-42.

Cartwright, E.K., Spicer, L., Smith, S.A., Lee, D., Fast, R., Paganini, S., Lawson, B.O., Nega, M., Easley, K., Schmitz, J.E., Bosinger, S.E., Paiardini, M., Chahroudi, A., Vanderford, T.H., Estes, J.D., Lifson, J.D., Derdeyn, C.A. Silvestri, G. (2016) CD8(+) Lymphocytes Are Required for Maintaining Viral Suppression in SIV-Infected Macaques Treated with Short-Term Antiretroviral Therapy. Immunity, 45: 656-68.

Li, S., Folkvord, J.M., Rakasz, E.G., Abdelaal, H.M., Wagstaff, R.K., Kovacs, K.J., Kim, H.O., Sawahata, R., Mawhinney, S., Masopust, D., Connick, E. Skinner, P.J. (2016) Simian Immunodeficiency Virus-Producing Cells in Follicles Are Partially Suppressed by CD8+ Cells In Vivo. J Virol, 90: 11168-80.

Saez-Cirion, A., Lacabaratz, C., Lambotte, O., Versmisse, P., Urrutia, A., Boufassa, F., BarreSinoussi, F., Delfraissy, J.F., Sinet, M., Pancino, G., Venet, A. Agence Nationale De Recherches Sur Le Sida, E.P.H.I.V.C.S.G. (2007) HIV controllers exhibit potent CD8 T cell capacity to suppress HIV infection ex vivo and peculiar cytotoxic T lymphocyte activation phenotype. Proc Natl Acad Sci U S A, 104: 6776-81.

\section{Figures}

\section{Figure 1. Preparation of JRCSF६nef virus pool}

(a) Scheme of the preparation of JRCSF $\xi n e f$ virus pool. The detailed procedure is summarized in Materials and Method section. Primers A-F denotes the location of pJRCSF. For the verification of mutations in the nef region, sequencing was performed at three steps ("Sequencings $A, B$ and $C$ "

This article is protected by copyright. All rights reserved. 
in the figure), and the results are shown in Supplemental Fig. 1-3 and Supplemental Table 1. (b) Preparation of JRCSF $\xi$ nef DNA fragment. The size of JRCSF $\xi$ nef DNA fragment is expected to be $\sim 9.4 \mathrm{~kb}$ when run in gel electrophoresis alongside the markers of HindllI digest of lambda DNA and 1kb DNA ladder. (c) Infectivity of WT JR-CSF and JRCSF $\xi$ nef virus pool. Viral infectivity was measured by TZM-bl assay and normalized to the amount of viral p24 antigen. (d) Number of mutations in the nef region of JRCSF $\xi$ nef virus pool. cDNA was synthesized from JRCSF $\xi$ nef virus pool and cloned into TOPO vectors for sequencing. 270 clones were sequenced and number of mutations per amplicon is shown. (e) A phylogenetic tree of the sequences of nef of the 270 clones from JRCSF $\xi$ nef virus pool. Parental (WT) virus is indicated by asterisk. A scale bar indicates 0.005 nucleotide substitutions per site.

a
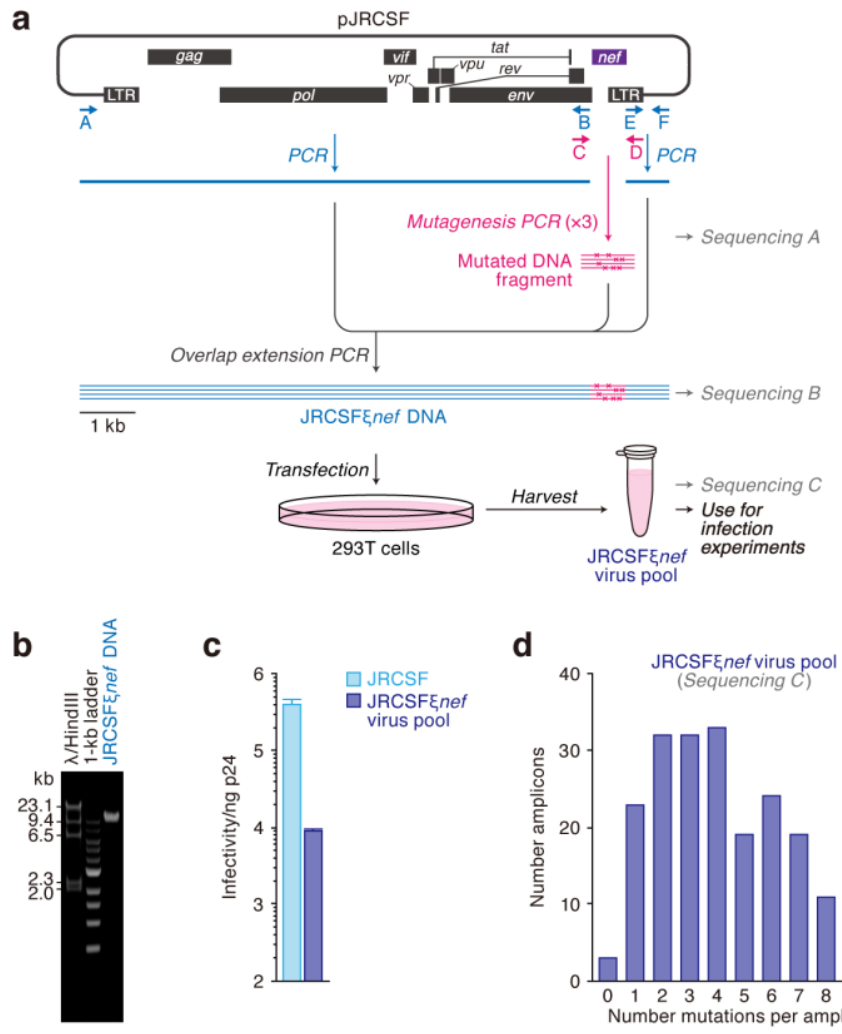

C

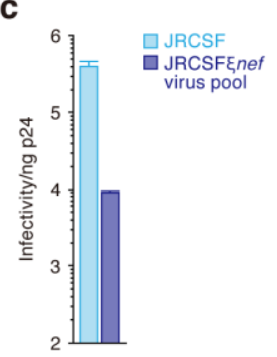

d

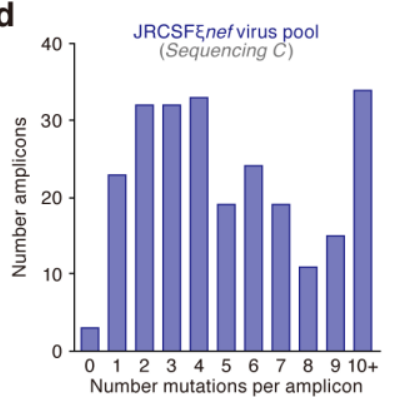

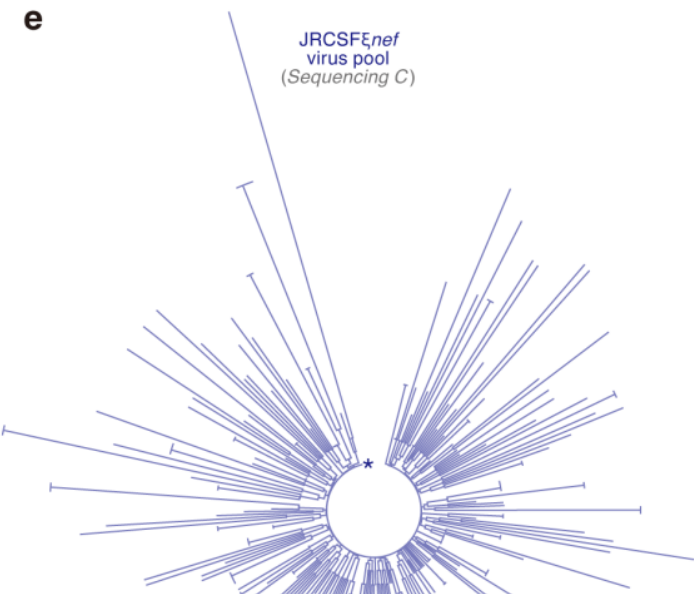

JRCSFEnef Sequencing $C$ 


\section{Figure 2. Stochastic HIV-1 infection in Jurkat-CCR5 cells}

(a) The growth of JRCSF $\xi$ nef virus pool in 4 independent Jurkat-CCR5 cell cultures (cultures A-D). (b) Phylogenetic trees of the nef region of JRCSF $\xi$ nef virus pool in in 4 independent Jurkat-CCR 5 cell cultures. The nef region of the viruses collected from the culture supernatant at days 6 (circle), 10 (triangle) and 14 (diamond) was sequenced. The sequences are aligned on a phylogeny tree relative to WT JRCSF nef sequence as a reference. The mutants dominantly detected are indicated by colors.
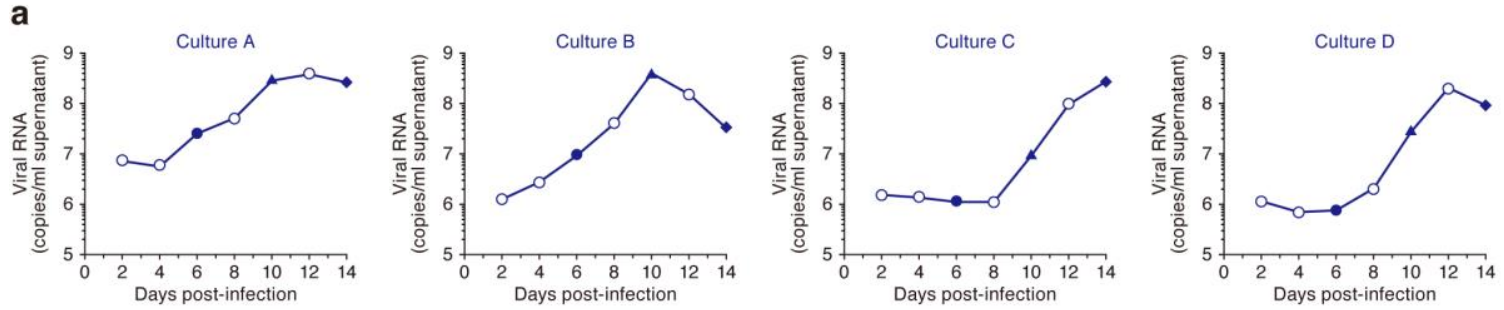

b
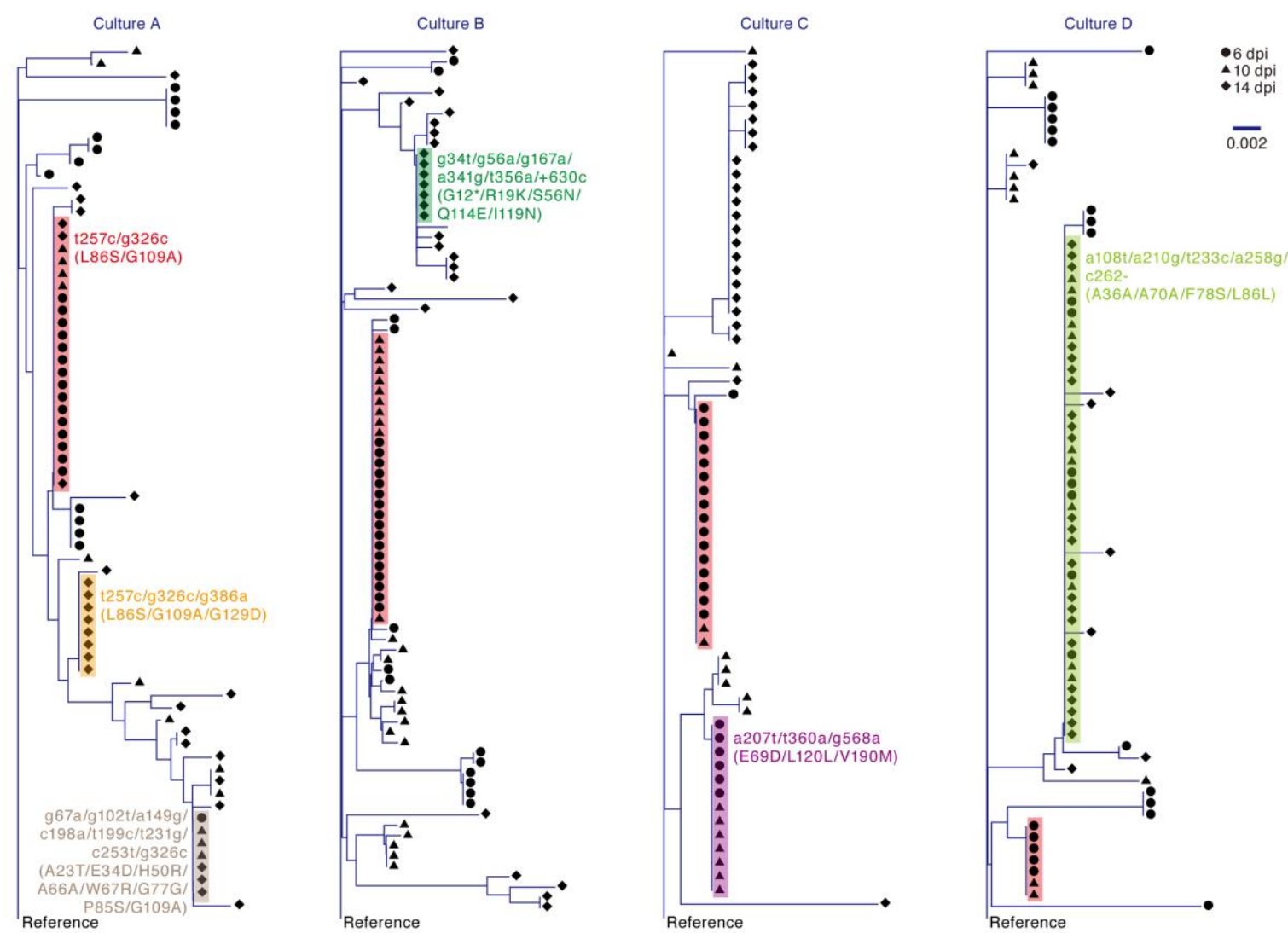

This article is protected by copyright. All rights reserved. 


\section{Figure 3. Infection of JRCSF६nef virus pool in humanized mice}

(a) Experimental setup. Three humanized mice were infected with JRCSF६nef virus pool. Blood was collected at 1, 2, 4, 6 wpi and mice were sacrificed at 9 wpi and organs indicated were collected. (b) (Left) The growth of JRCSF nef virus pool $(n=3)$ and WT JRCSF $(n=5)$. The amounts of viral RNA in the three mice infected with JRCSF $\xi$ nef virus pool are individually indicated by lines, and the averages of viral RNA in WT JRCSF-infected mice $(n=5)$ are shown with SEM. (Right) The area under the curve (AUC) of the viral RNA. Each dot indicates the result from individual infected mouse.

(c) (Left) Decrease of peripheral CD4 ${ }^{+} \mathrm{T}$ cells. The fold change of the level of peripheral CD4 ${ }^{+} \mathrm{T}$ cells in the three mice infected with JRCSF $\xi$ nef virus pool are individually indicated by lines, and the averages of the fold change of the level of peripheral CD4 ${ }^{+}$T cells in WT JRCSF-infected mice $(n=5)$ and mockinfected mice $(n=6)$ are shown with SEM. (Right) The AUC of the fold change of the level of peripheral CD4 ${ }^{+} \mathrm{T}$ cells. Each dot indicates the result from each individual infected mouse. (d) Mutations detected in each organ of the mice

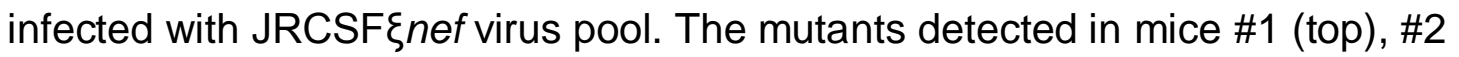
(middle) and \#3 (bottom) are summarized in pie charts. The numbers in parentheses indicate the numbers of sequences analyzed. Each color indicates the mutant detected, and "* $X$ " are the mutants that contain more than five mutations. The list of mutants detected is summarized in Supplemental Table 2. 
a

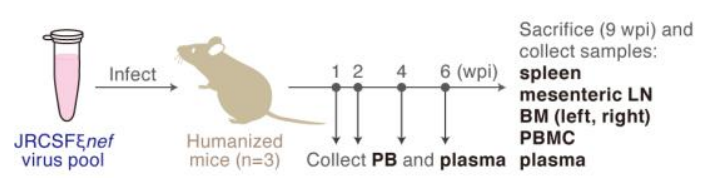

b

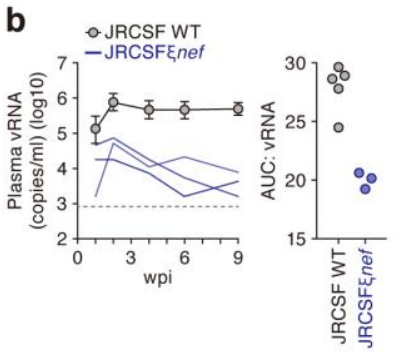

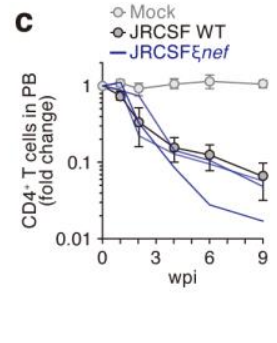

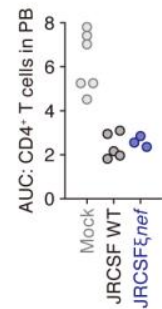

d
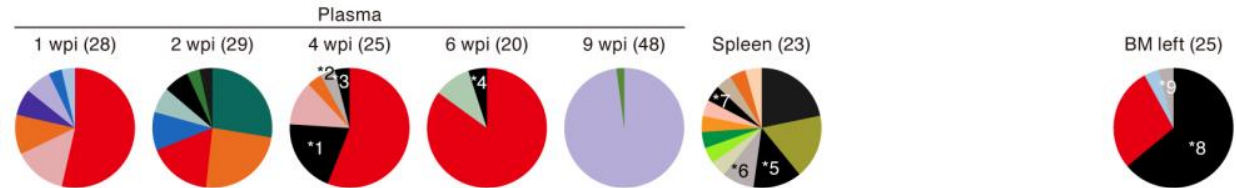

PBL (34) Mouse
$\# 1$
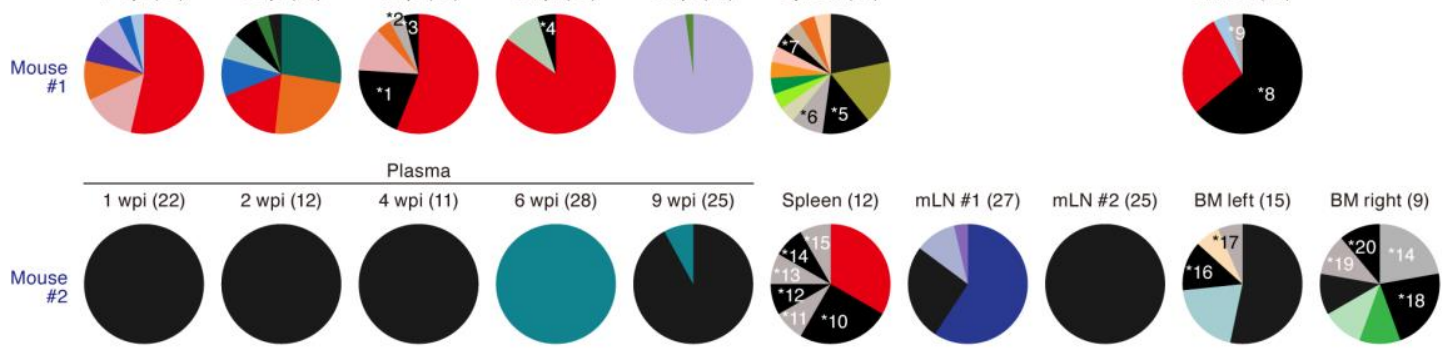

PBL (5)
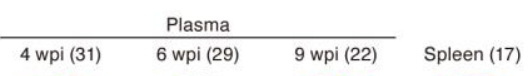

BM left (11)

BM right (12)

Mouse
$\# 3$
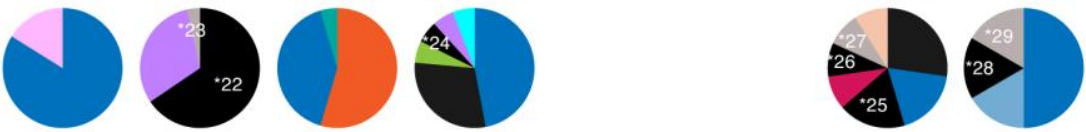

- WT (parental)
- $\mathrm{L} 86 \mathrm{~S} / \mathrm{G} 109 \mathrm{~A}$
E69D/V190M
W5G
W5G/E211G
W78S/P88A
- E69D/L120Q/D121Y/V190M
- F78S/P88/G109A
- G109A
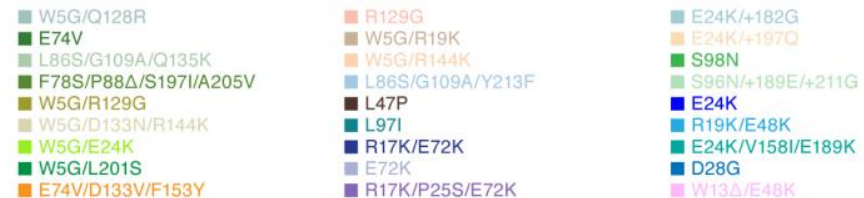

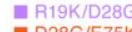
D28G/E75K - W5G/R19K/R311 D28G/E165K - D28G/E74K

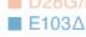

Figure 4. VOA showing at least two replication-competent mutated viruses (W5G and D28G) in the spleen of humanized mice

(a) A scheme of VOA. (b and c) Pie charts of the viruses rescued from the spleens of mouse \#1 (b) and mouse \#3 (c).

a

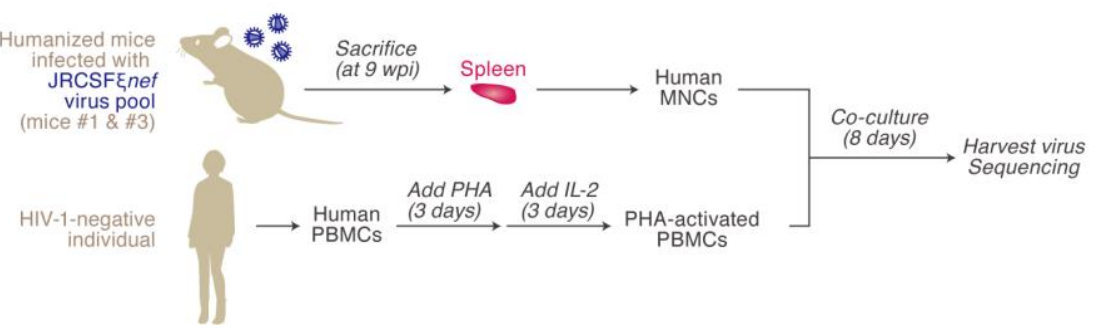

b

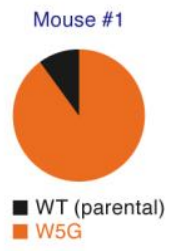

c

Mouse \#3

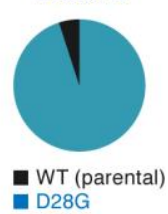

This article is protected by copyright. All rights reserved. 